\title{
Invariance of Conjunctions of Polynomial Equalities for Algebraic Differential Equations ${ }^{\star}$
}

\author{
Khalil Ghorbal $^{1}$, Andrew Sogokon ${ }^{2}$, and André Platzer ${ }^{1}$ \\ 1 Carnegie Mellon University, Computer Science Department, Pittsburgh, PA, USA \\ $\{$ kghorbal |aplatzer\}@es. cmu.edu \\ ${ }^{2}$ University of Edinburgh, LFCS, School of Informatics, Edinburgh, Scotland, UK \\ a.sogokonesms.ed.ac.uk
}

\begin{abstract}
In this paper we seek to provide greater automation for formal deductive verification tools working with continuous and hybrid dynamical systems. We present an efficient procedure to check invariance of conjunctions of polynomial equalities under the flow of polynomial ordinary differential equations. The procedure is based on a necessary and sufficient condition that characterizes invariant conjunctions of polynomial equalities. We contrast this approach to an alternative one which combines fast and sufficient (but not necessary) conditions using differential cuts for soundly restricting the system evolution domain.
\end{abstract}

\section{Introduction}

The problem of reasoning about invariant sets of dynamical systems is of fundamental importance to verification and modern control design [3|22|28|26]. A set is an invariant of a dynamical system if no trajectory can escape from it. Of particular interest are safety assertions that describe states of the system which are deemed safe; it is clearly important to ensure that these sets are indeed invariant.

Hybrid systems combine discrete and continuous behavior and have found application in modelling a vast quantity of industrially relevant designs, many of which are safety-critical. In order to verify safety properties in hybrid models, one often requires the means of reasoning about safety in continuous systems. This paper focuses on developing and improving the automation of reasoning principles for a particular class of invariant assertions for continuous systems - conjunctions of polynomial equalities; these can be used, e.g. to assert the property that certain values (temperature, pressure, water level, etc.) in the system are maintained at a constant level as the system evolves.

In practice, it is highly desirable to have the means of deciding whether a given set is invariant in a particular dynamical system. It is equally important that such methods be efficient enough to be of practical utility. This paper seeks to address both of these issues. The contributions of this paper are twofold:

- It extends differential radical invariants [11] to obtain a characterization of invariance for algebraic sets under the flow of algebraic differential equations. It also

\footnotetext{
* This material is based upon work supported by the National Science Foundation by NSF CAREER Award CNS-1054246, NSF EXPEDITION CNS-0926181, CNS-0931985, DARPA FA8750-12-2-0291 and EPSRC EP/I010335/1.
}

(C) Springer International Publishing Switzerland 2014 Markus Müller-Olm and Helmut Seidl (Eds.): SAS-21, LNCS 8723, pp. 151-167, 2014 DOI: 10.1007/978-3-319-10936-7_10 
introduces an optimized decision procedure to decide the invariance of algebraic sets.

- It explores an approach combining deductively less powerful rules [15|27|17|25] using differential cuts [23] to exploit the structure of the system to yield efficient proofs even for non-polynomial systems. Furthermore, differential cuts [23] are shown to fundamentally improve the deductive power of Lie's criterion [15].

The two approaches to proving invariance of conjunctive equational assertions explored in this paper are complementary and aim at improving proof automation-deductive power and efficiency-in deductive formal verification tools. The detailed proofs of all presented results are available in [12].

Content. In Section2, we recall some basic definitions and concepts. Section 3 introduces a new proof rule to check the invariance of a conjunction of polynomial equations along with an optimized implementation. Section 4 presents another novel approach to check invariance of a conjunction; it leverages efficient existing proof rules together with differential cuts and differential weakening. An automated proof strategy that builds on top of this idea is given in Section 5 . The average performance of these different approaches is assessed using a set of 32 benchmarks (Section6).

\section{Preliminaries}

Let $\boldsymbol{x}=\left(x_{1}, \ldots, x_{n}\right): \mathbb{R}^{n}$, and $\boldsymbol{x}(t)=\left(x_{1}(t), \ldots, x_{n}(t)\right)$, where $x_{i}: \mathbb{R} \rightarrow \mathbb{R}$, $t \mapsto x_{i}(t)$. The ring of polynomials over the reals will be denoted by $\mathbb{R}\left[x_{1}, \ldots, x_{n}\right]$. We consider autonomous 3 differential equations described by polynomial vector fields.

Definition 1 (Polynomial Vector Field). Let $p_{i}, 1 \leq i \leq n$, be multivariate polynomials in the polynomial ring $\mathbb{R}[\boldsymbol{x}]$. A polynomial vector field, $\boldsymbol{p}$, is an explicit system of ordinary differential equations with polynomial right-hand side:

$$
\frac{d x_{i}}{d t}=\dot{x}_{i}=p_{i}(\boldsymbol{x}), \quad 1 \leq i \leq n .
$$

One important problem is that of checking the invariance of a variety (or algebraic set), with evolution domain constraints $H$; that is, we ask whether a polynomial conjunction $h_{1}=0 \wedge \cdots \wedge h_{r}=0$, initially true, holds true in all reachable states that satisfy the evolution domain constraints. The problem is equivalent to the validity of the following formula in differential dynamic logic [22]:

$$
\left(h_{1}=0 \wedge \cdots \wedge h_{r}=0\right) \rightarrow[\dot{\boldsymbol{x}}=\boldsymbol{p} \& H]\left(h_{1}=0 \wedge \cdots \wedge h_{r}=0\right)
$$

where $[\dot{\boldsymbol{x}}=\boldsymbol{p} \& H] \psi$ is true in a state $\boldsymbol{x}_{\iota}$ if the postcondition $\psi$ is true in all states reachable from $\boldsymbol{x}_{\iota}$-satisfying $H$-by following the differential equation $\dot{\boldsymbol{x}}=\boldsymbol{p}$ for any amount of time as long as $H$ is not violated. For simplicity, for a polynomial $h$ in $\boldsymbol{x}$, we write $h=0$ for $h(\boldsymbol{x})=0$.

\footnotetext{
${ }^{3}$ Autonomous means that the rate of change of the system over time depends only on the system's state, not on time. Non-autonomous systems with time dependence can be made autonomous by adding a new state variable to account for the progress of time.
} 
Geometrically, the dL formula in Eq. (2) is true if and only if the solution $x(t)$ of the initial value problem $\left(\dot{\boldsymbol{x}}=\boldsymbol{p}, \boldsymbol{x}(0)=\boldsymbol{x}_{\iota}\right)$, with $h_{i}\left(\boldsymbol{x}_{\iota}\right)=0$ for $i=1, \ldots, r$, is a real root of the system $h_{1}=0, \ldots, h_{r}=0$ as long as it satisfies the constraints $H$.

The algebraic counterpart of varieties are ideals. Ideals are sets of polynomials that are closed under addition and external multiplication. That is, if $I$ is an ideal, then for all $h_{1}, h_{2} \in I$, the sum $h_{1}+h_{2} \in I$; and if $h \in I$, then, $q h \in I$, for all $q \in \mathbb{R}\left[x_{1} \ldots, x_{n}\right]$.

We will use $\nabla h$ to denote the gradient of a polynomial $h$, that is the vector of its partial derivatives $\left(\frac{\partial h}{\partial x_{1}}, \ldots, \frac{\partial h}{\partial x_{n}}\right)$. The Lie derivative of a polynomial $h$ along a vector field $\boldsymbol{p}$ is defined as follows (the symbol "." denotes the scalar product):

$$
\mathfrak{L}_{\boldsymbol{p}}(h) \stackrel{\text { def }}{=} \nabla h \cdot \boldsymbol{p}=\sum_{i=1}^{n} \frac{\partial h}{\partial x_{i}} p_{i} .
$$

Higher-order Lie derivatives are: $\mathfrak{L}_{\boldsymbol{p}}^{(k+1)}(h)=\mathfrak{L}_{\boldsymbol{p}}\left(\mathfrak{L}_{\boldsymbol{p}}^{(k)}(h)\right)$, where $\mathfrak{L}_{\boldsymbol{p}}^{(0)}(h)=h$.

\section{Characterizing Invariance of Conjunctive Equations}

In this section we give an exact characterization of invariance for conjunctions of polynomial equalities under the flow of algebraic differential equations. The characterization, as well as the proof rule, generalize our previous work which handles purely equational invariants of the form $h=0$ without considering evolution domains.

The differential radical invariants proof rule DRI [11, Theorem 2] has been shown to be a necessary and sufficient criterion for the invariance of equations of the form $h=0$ :

$$
\text { (DRI) } \frac{h=0 \rightarrow \bigwedge_{i=0}^{N-1} \mathfrak{L}_{\boldsymbol{p}}^{(i)}(h)=0}{h=0 \rightarrow[\dot{\boldsymbol{x}}=\boldsymbol{p}] h=0} .
$$

The order $N \geq 1$ denotes the length of the chain of ideals $\langle h\rangle \subseteq\left\langle h, \mathfrak{L}_{p}(h)\right\rangle \subseteq \cdots$ which reaches a fixed point after finitely many steps by the ascending chain property of Noetherian rings. Thus, the order $N$ is always finite and computable-using Göbner Bases [4] - for polynomials with rational coefficients. The premise of the proof rule DRI is a real quantifier elimination problem and can be solved algorithmically [5].

A naïve approach to prove invariance of a conjunction $h_{1}=0 \wedge \cdots \wedge h_{r}=0$, without evolution domain constraints, is to use the proof rule DRI together with the following sum-of-squares equivalence from real arithmetic:

$$
h_{1}=0 \wedge \cdots \wedge h_{r}=0 \equiv_{\mathbb{R}} \sum_{i=1}^{r} h_{i}^{2}=0 .
$$

Sums-of-squares come at the price of doubling the polynomial degree, thereby increasing the complexity of checking the premise (Section 3.2 discusses the link between polynomial degree and the complexity of DRI-based proof rules). Instead, we present an extension of the proof rule DRI that exploits the underlying logical structure of conjunctions. For a conjunction of equations $h_{1}=0 \wedge \cdots \wedge h_{r}=0$, the order $N$ is generalized to the length of the chain of ideals formed by all the polynomials $h_{1}, \ldots, h_{r}$ 
and their successive Lie derivatives:

$$
I=\left\langle h_{1}, \ldots, h_{r}\right\rangle \subseteq\left\langle h_{1}, \ldots, h_{r}, \mathfrak{L}_{\boldsymbol{p}}\left(h_{1}\right), \ldots, \mathfrak{L}_{\boldsymbol{p}}\left(h_{r}\right)\right\rangle \subseteq\left\langle h_{1}, \ldots, \mathfrak{L}_{\boldsymbol{p}}^{(2)}\left(h_{r}\right)\right\rangle \ldots
$$

Theorem 2 (Conjunctive Differential Radical Characterization). Let $h_{1}, \ldots, h_{r} \in$ $\mathbb{R}[\boldsymbol{x}]$ and let $H$ denote some topologically open evolution domain constraint. Then, the conjunction $h_{1}=0 \wedge \cdots \wedge h_{r}=0$, is invariant under the flow of the vector field $\boldsymbol{p}$, subject to the evolution constraint $H$, if and only if

$$
H \vdash \bigwedge_{j=1}^{r} h_{j}=0 \rightarrow \bigwedge_{j=1}^{r} \bigwedge_{i=1}^{N-1} \mathfrak{L}_{p}^{(i)}\left(h_{j}\right)=0 .
$$

where $N$ denotes the order of the conjunction.

Here $\vdash$ is used, as in sequent calculus, to assert that whenever the constraint $H$ (antecedent) is satisfied, then at least one (in this case, the only) formula to the right of $\vdash$ is also true. The proof is in the companion report [12]. When the evolution domain constraints are dropped ( $H=$ True) and $r=1$ (one equation), one recovers exactly the statement of [11, Theorem 2] which characterizes invariance of atomic equations. Intuitively, Theorem 2 says that on the invariant algebraic set, all higher-order Lie derivatives of each polynomial $h_{i}$ must vanish. It adds however a crucial detail: checking finitely many-exactly $N$-higher-order Lie derivatives is both necessary and sufficient. The theorem does not check for invariance of each conjunct taken separately, rather it handles the conjunction simultaneously. The order $N$ is a property of the ideal chain formed by all the polynomials and their Lie derivatives. If $N_{i}$ denotes the order of each atom $h_{i}$ taken separately, then one can readily see that

$$
N \leq \max _{i} N_{i} .
$$

The equality does not hold in general: consider for instance $h_{1}=x_{1}, h_{2}=x_{2}$ and $\boldsymbol{p}=\left(x_{2}, x_{1}\right)$. Since $\mathfrak{L}_{\boldsymbol{p}}^{(2)}\left(h_{i}\right)=h_{i}$, for $i=1,2$, we have $N_{1}=N_{2}=2$. However,

$$
\left\langle x_{1}, x_{2}\right\rangle=\left\langle h_{1}, h_{2}\right\rangle \subseteq\left\langle h_{1}, h_{2}, \mathfrak{L}_{\boldsymbol{p}}\left(h_{1}\right), \mathfrak{L}_{\boldsymbol{p}}\left(h_{2}\right)\right\rangle=\left\langle x_{1}, x_{2}, x_{2}, x_{1}\right\rangle=\left\langle x_{1}, x_{2}\right\rangle,
$$

which means that $N=1$. This example highlights one of the main differences between this work and the characterization given in [16, Theorem 24], where the criterion is given by

$$
H \vdash \bigwedge_{j=1}^{r} h_{j}=0 \rightarrow \bigwedge_{j=1}^{r} \bigwedge_{i=1}^{N_{j}-1} \mathfrak{L}_{p}^{(i)}\left(h_{j}\right)=0 .
$$

The computation of each order $N_{j}$ requires solving $N_{j}$ ideal membership problems. One can appreciate the difference with the criterion of Theorem 2 which only requires $N$ ideal membership checks for the entire conjunction. In the worst case, when $N=$ $N_{k}=\max _{i} N_{i}$, Theorem 2 performs $\sum_{j=1, j \neq k}^{r} N_{j}$ fewer ideal membership checks compared to the criterion of Eq. (9). A smaller order $N$ confers an additional benefit of reducing the cost of quantifier elimination-discussed in Section 3.2 - by bringing down both the total number of polynomials and their maximum degree. 
Remark 3. The order $N$ in Theorem 2 can be reduced further at the prohibitive $\cos 4^{4}$ of computing the real radicals of the ideals in Eq. (6). Ideally, one should also account for $H$ when computing $N$. When $H$ is an algebraic set, its generators should be appended to the ideal $\left\langle h_{1}, \ldots, h_{r}\right\rangle$. We leave the semi-algebraic case for future work.

Using Theorem 2, the differential radical invariant proof rule DRI [11] generalizes to conjunctions of equations with evolution domain constraints as follows:

$$
\left(\mathrm{DRI}_{\wedge}\right) \frac{H \vdash\left(\bigwedge_{j=1}^{r} h_{j}=0\right) \rightarrow \bigwedge_{j=1}^{r} \bigwedge_{i=1}^{N-1} \mathfrak{L}_{p}^{(i)}\left(h_{j}\right)=0}{\left(\bigwedge_{j=1}^{r} h_{j}=0\right) \rightarrow[\dot{\boldsymbol{x}}=\boldsymbol{p} \& H]\left(\bigwedge_{j=1}^{r} h_{j}=0\right)} .
$$

Next, we implement the proof rule $\mathrm{DRI}_{\wedge}$ and discuss its theoretical complexity.

\subsection{Decision Procedure}

To check the validity of the premise in the proof rule $\mathrm{DRI}_{\wedge}$, one needs to compute the order $N$ and to decide a purely universally quantified sentence in the theory of real arithmetic. These two tasks do not have to be performed in that precise order. We present an algorithm that computes $N$ on the fly while breaking down the quantifier elimination problem into simpler sub-problems.

Algorithm 1 implements the proof rule $\mathrm{DRI}_{\wedge}$. The algorithm returns True if and only if the candidate is an invariant. The variable $\check{N}$ strictly increases and converges, from below, toward the finite unknown order $N$. It is therefore a decision procedure for the invariance problem with conjunctive equational candidates.

At each iteration of the while loop it checks whether a fixed point of the chain of ideals has been reached, implying $\check{N}=N$. To this end, it computes a Gröbner basis (GB) of the ideal I (line 2), containing the polynomials $h_{i}$ as well as their respective higher-order Lie derivatives up to the derivation order $\check{N}-1$. Then it enters a foreach loop (line 8), where it computes the $\check{N}$ th order Lie derivatives and their respective reductions (or remainders) (LieD) by the Gröbner basis GB. All Lie derivatives with non-zero remainders are stored in the list LD (line 12). If the list is empty, then all $\check{N}$ th Lie derivatives are in the ideal I: the fixed point of the chain of ideals is reached, and $\check{N}=N$. This also means that True can be returned since all prior quantifier elimination calls returned True. Otherwise, the outermost while loop (line 5) needs to be executed one more time after increasing $\check{N}$ (line 20). Before re-executing the while loop, however, we make sure that the premise of the proof rule $\mathrm{DRI}_{\wedge}$ holds up to $\check{N}$. Since in this case, we know that $\check{N}<N$, if the quantifier elimination fails to discharge the premise of the proof rule $\mathrm{DRI}_{\wedge}$ at $\check{N}$, then we do not need to go any further as the invariance property is already falsified.

The while loop decomposes the right hand side of the implication in Eq. 10] along the conjunction $\bigwedge_{i=1}^{N-1}$ : the $i$ th iteration checks whether the conjunction $\bigwedge_{j=1}^{r} \mathfrak{L}_{p}^{(i)} h_{j}$ vanishes. The main purpose of the foreach loop in line 16 is to decompose further the

\footnotetext{
${ }^{4}$ The upper bound on the degrees of the generators of the real radical of an ideal $I$ is $d^{2}{ }^{O\left(n^{2}\right)}$
} [20], where $d$ is the maximum degree of the generators of $I$. 


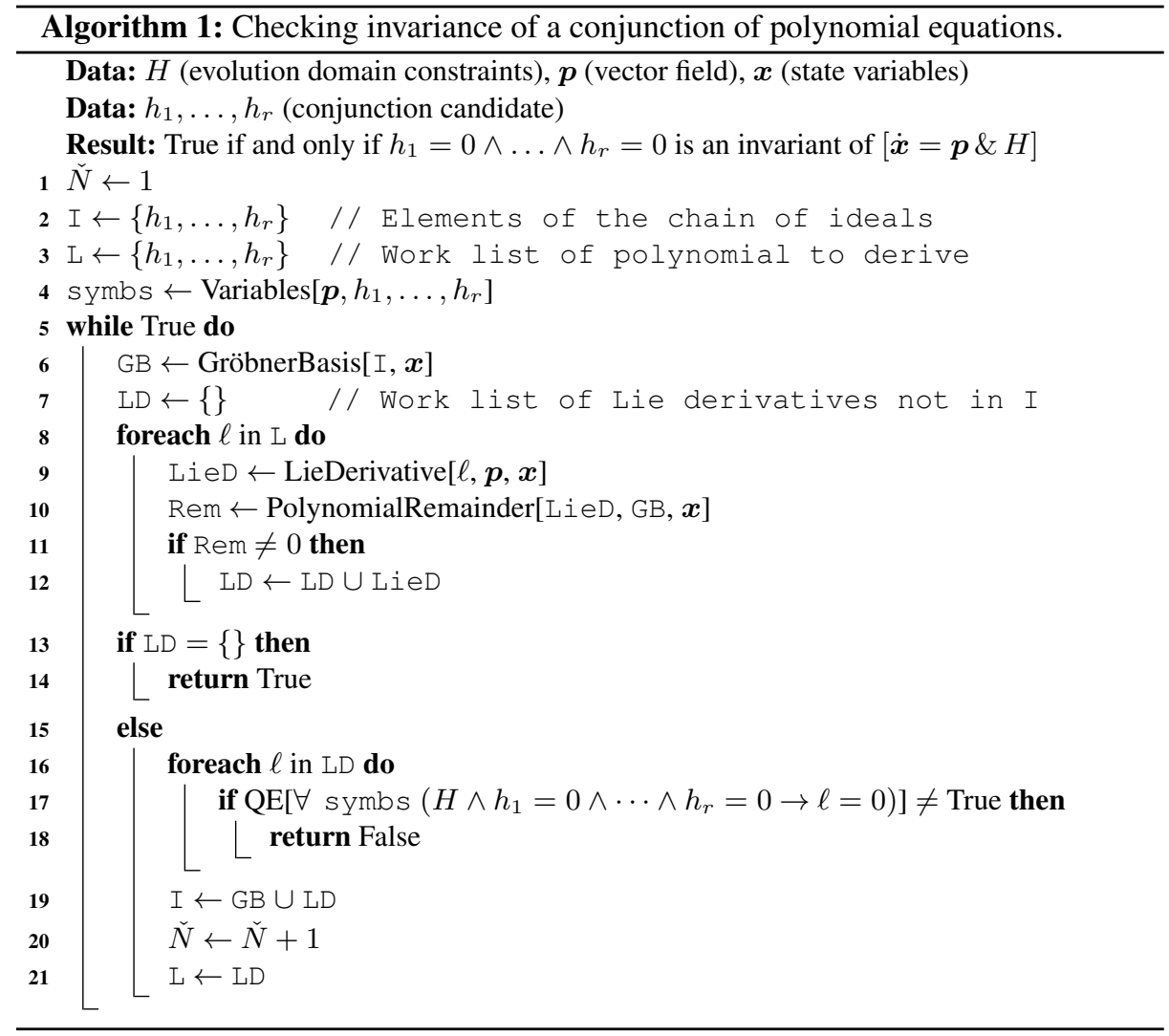

conjunction $\bigwedge_{j=1}^{r}$ using the logical equivalence $a \rightarrow(b \wedge c) \equiv(a \rightarrow b) \wedge(a \rightarrow c)$ for any boolean variables $a, b$, and $c$. This leads to more tractable problems of the form:

$$
H \vdash \bigwedge_{j=1}^{r} h_{j}=0 \rightarrow \mathfrak{L}_{\boldsymbol{p}}^{(i)}\left(h_{j}\right)=0 .
$$

Observe that the quantifier elimination problem in line 17 performs a universal closure for all involved symbols - state variables and parameters - denoted by symbs and determined once at the beginning of the algorithm using the procedure variables (line 4). Besides, the quantifier elimination problem in line 17 can be readily adapted to explicitly return extra conditions on the parameters to ensure invariance of the given conjunction. When the algorithm returns False, any counterexample to the quantifier elimination problem of line 17 can be used as an initial condition for a concrete counterexample that falsifies the invariant. 


\subsection{Complexity}

Algorithm 1 relies on two expensive procedures: deciding purely universally quantified sentences in the theory of real arithmetic (line 17p) and ideal membership of multivariate polynomials using Gröbner bases (line 6). We discuss their respective complexity.

Quantifier elimination over the reals is decidable [29]. The purely existential fragment of the theory of real arithmetic has been shown to exhibit singly exponential time complexity in the number of variables [1]. Theoretically, the best bound on the complexity of deciding a sentence in the existential theory of $\mathbb{R}$ is given by $(s d)^{O(n)}$, where $s$ is the number of polynomials in the formula, $d$ their maximum degree and $n$ the number of variables [1]. However, in practice this has not yet led to an efficient decision procedure, so typically it is much more efficient to use partial cylindrical algebraic decomposition (PCAD) due to Collins \& Hong [5], which has running time complexity doubly-exponential in the number of variables.

Ideal membership of multivariate polynomials with rational coefficients is complete for EXP SPACE [18]. Gröbner bases [4] allow membership checks in ideals generated by multivariate polynomials. Significant advances have been made for computing Gröbner bases [9]10] which in practice can be expected to perform very well. The degree of the polynomials involved in a Gröbner basis computation can be very large. Theoreti-

cally, a Gröbner basis may contain polynomials with degree $2^{2^{d}}$ [19]. The degrees of all the polynomials involved are bounded by $O\left(d^{2^{n}}\right)$ [8]. Gröbner bases are also highly sensitive to the monomial order arranging the different monomials of a multivariate polynomial (see, e.g., [6, Chapter 2] for formal definitions). The Degree Reverse Lexicographic (degrevlex) order gives (on average) Gröbner bases with the smallest total degree [2], although there exist known examples (cf. Mora's example in [14]) for which, even for the degrevlex monomial ordering, the (reduced) Gröbner basis contains a polynomial of total degree $O\left(d^{2}\right)$. Finally, the rational coefficients of the generators of Gröbner bases may become involved (compared to the rational coefficients of the original generators of the ideal), which can have a negative impact on the running time and memory requirements.

\subsection{Optimization}

The theoretical complexity of both the quantifier elimination and Gröbner bases algorithms suggests several opportunities for optimization for Algorithm 1 . The maximal degree of the polynomials appearing in $H$ is assumed to be fixed. We can reduce the polynomial degrees in the right-hand side of the implication in Eq. (11) as follows: by choosing a total degree monomial ordering (e.g. degrevlex), the remainder Rem has at most the same total degree as LieD; replacing LieD by Rem serves to reduce (on average) the cost of calling a quantifier elimination procedure. Lem. 4 proves that substituting LieD by its remainder Rem in line 17 does not compromise correctness.

Lemma 4. Let $q$ be the remainder of the reduction of the polynomial s by the Gröbner basis of the ideal generated by the polynomials $h_{1}, \ldots, h_{r}$. Then,

$$
h_{1}=0 \wedge \cdots \wedge h_{r}=0 \rightarrow s=0 \text { if and only if } h_{1}=0 \wedge \cdots \wedge h_{r}=0 \rightarrow q=0 .
$$


The same substitution reduces the Gröbner basis computation cost since it attempts to keep a low maximal degree in all the polynomials appearing in the generators of the ideal I. Lem. 5 shows that it is safe to perform this substitution: the ideal I remains unchanged regardless of whether we choose to construct the list LD using LieD or Rem.

Lemma 5. Let $q$ be the remainder of the reduction of the polynomial s by the Gröbner basis of the ideal generated by the polynomials $h_{1}, \ldots, h_{r}$. Then,

$$
\left\langle h_{1}, \ldots, h_{r}, s\right\rangle=\left\langle h_{1}, \ldots, h_{r}, q\right\rangle .
$$

Although this optimization reduces the total degree of the polynomials involved, the coefficients of the remainder $q$ may get more involved than the coefficients of the original polynomial $s$. In [12], we give an example featuring the Motzkin polynomial where such problem occurs. In Section 6 we give an empirical comparison of the optimizedas detailed in this section-versus the unoptimized version of Algorithm 1 .

\section{Sufficient Conditions for Invariance of Equations}

The previous section dealt with a method for proving invariance which is both necessary and sufficient for conjunctions of polynomial equalities. Given the proof rule $\mathrm{DRI}_{\wedge}$, it is natural to ask whether previously proposed sufficient proof rules are still relevant. After all, theoretically, $\mathrm{DRI}_{\wedge}$ is all that is required for producing proofs of invariance in this class of problems. This is a perfectly legitimate question; however, given the complexity of the underlying decision procedures needed for $\mathrm{DRI}_{\wedge}$ it is perhaps not surprising that one will eventually face scalability issues. This, in turn, motivates a different question - can one use proof rules (which are perhaps deductively weaker than $\mathrm{DRI}_{\wedge}$ ) in such a way as to attain more computationally efficient proofs of invariance?

Before addressing this question, this section will review existing sufficient proof rules which allow reasoning about invariance of atomic equational assertions. In Fig. 11, $\mathrm{DI}_{=}$shows the equational differential invariant [23] proof rule. The condition is sufficient (but not necessary) and characterizes polynomial invariant functions [23 25]. The premise of the Polynomial-consecution rule [27|17], P-c in Fig.11. requires $\mathfrak{L}_{p}(h)$ to be in the ideal generated by $h$. This condition is also only sufficient and was mentioned as early as 1878 [7]. The Lie proof rule gives Lie's criterion [15|21|25] for invariance of $h=0$ and characterizes smooth invariant manifolds. The rule DW is called differential weakening [24] and covers the trivial case when the evolution constraint implies the invariant candidate; in contrast to all other rules in the table, DW can work with arbitrary invariant assertions.

Unlike the necessary and sufficient condition provided by the rule DRI (see Eq. (4)), all the other proof rules in Figure 1 only impose sufficient conditions and may thus fail at a proof even in cases when the candidate is indeed an invariant.

The purpose of all the rules shown in Figure 1, save perhaps DW, is to show invariance of atomic equations. However, in general, one faces the problem $F \rightarrow[\dot{\boldsymbol{x}}=$ $\boldsymbol{p} \& H] C$, where $F$ is a formula defining a set of states where the system is initialized, and $C$ is the post-condition where the system always enters after following the differential equation $\dot{\boldsymbol{x}}=\boldsymbol{p}$ as long as the domain constraint $H$ is satisfied. 


$$
\begin{array}{cc}
\left(\mathrm{DI}_{=}\right) \frac{H \vdash \mathfrak{L}_{\boldsymbol{p}}(h)=0}{(h=0) \rightarrow[\dot{\boldsymbol{x}}=\boldsymbol{p} \& H](h=0)} & (\mathrm{P}-\mathrm{c}) \frac{H \vdash \mathfrak{L}_{\boldsymbol{p}}(h) \in\langle h\rangle}{(h=0) \rightarrow[\dot{\boldsymbol{x}}=\boldsymbol{p} \& H](h=0)} \\
(\mathrm{Lie}) \frac{H \vdash h=0 \rightarrow\left(\mathfrak{L}_{\boldsymbol{p}}(h)=0 \wedge \nabla h \neq \mathbf{0}\right)}{(h=0) \rightarrow[\dot{\boldsymbol{x}}=\boldsymbol{p} \& H](h=0)} & (\mathrm{DW}) \frac{H \vdash F}{F \rightarrow[\dot{\boldsymbol{x}}=\boldsymbol{p} \& H] F}
\end{array}
$$

Fig. 1: Proof rules for checking the invariance of $h=0$ w.r.t. the vector field $\boldsymbol{p}$ : $\mathrm{DI}=[25$, Theorem 3], P-c [27]. Lemma 2], Lie [21, Theorem 2.8], DW [24, Lemma 3.6]

One way to prove such a statement is to find an invariant $I$ which is true initially (i.e. $F \rightarrow I)$, is indeed an invariant for the system $(I \rightarrow[\dot{\boldsymbol{x}}=\boldsymbol{p} \& H] I)$, and implies the post-condition $(I \rightarrow C)$. These conditions can be formalized in the proof rule [26]

$$
(\operatorname{Inv}) \frac{F \rightarrow I \quad I \rightarrow[\dot{\boldsymbol{x}}=\boldsymbol{p} \& H] I \quad I \rightarrow C}{F \rightarrow[\dot{\boldsymbol{x}}=\boldsymbol{p} \& H] C} .
$$

In this paper we consider the special case when the invariant is the same as the postcondition, so we can drop the last clause and the rule becomes

$$
(\operatorname{Inv}) \frac{F \rightarrow C \quad C \rightarrow[\dot{\boldsymbol{x}}=\boldsymbol{p} \& H] C}{F \rightarrow[\dot{\boldsymbol{x}}=\boldsymbol{p} \& H] C} .
$$

In the following sections, we will be working in a proof calculus, rather than considering a single proof rule, and will call upon this definition in the proofs we construct.

\section{Differential Cuts and Lie's Rule}

When considering a conjunctive invariant candidate $h_{1}=0 \wedge h_{2}=0 \wedge \cdots \wedge h_{r}=0$, it may be the case that each conjunct considered separately is an invariant for the system. Then, one could simply invoke the following basic result about invariant sets to prove invariance of each atomic formula individually.

Proposition 6. Let $S_{1}, S_{2} \subseteq \mathbb{R}^{n}$ be invariant sets for the differential equation $\dot{\boldsymbol{x}}=\boldsymbol{p}$, then the set $S_{1} \cap S_{2}$ is also an invariant.

Corollary 7. The proof rule

$$
\left(\wedge_{\text {Inv }}\right) \frac{h_{1}=0 \rightarrow[\dot{\boldsymbol{x}}=\boldsymbol{p} \& H] h_{1}=0 \quad h_{2}=0 \rightarrow[\dot{\boldsymbol{x}}=\boldsymbol{p} \& H] h_{2}=0}{h_{1}=0 \wedge h_{2}=0 \rightarrow[\dot{\boldsymbol{x}}=\boldsymbol{p} \& H]\left(h_{1}=0 \wedge h_{2}=0\right)}
$$

is sound and may be generalized to accommodate arbitrarily many conjuncts.

Of course, one still needs to choose an appropriate proof rule from Figure 1 (or DRI) in order to prove invariance of atomic equational formulas. For purely polynomial problems it would be natural to attempt a proof using DRI first, but in the presence of transcendental functions, one may need to resort to other rules. In general however, even if the conjunction defines an invariant set, the individual conjuncts need not themselves be invariants. If such is the case, one cannot simply break down the conjunctive 
assertion using the rule $\wedge_{\text {Inv }}$ and prove invariance of each conjunct individually. In this section, we explore using a proof rule called differential cut (DC) to address this issue.

Differential cuts were introduced as a fundamental proof principle for differential equations [23] and can be used to (soundly) strengthen assumptions about the system evolution.

Proposition 8 (Differential Cut [23]). The proof rule

$$
\text { (DC) } \frac{F \rightarrow[\dot{\boldsymbol{x}}=\boldsymbol{p}] C \quad F \rightarrow[\dot{\boldsymbol{x}}=\boldsymbol{p} \& C] F}{F \rightarrow[\dot{\boldsymbol{x}}=\boldsymbol{p}] F},
$$

where $C$ and $F$ denote quantifier-free first-order formulas, is sound.

Remark 9. The rule $\wedge_{\text {Inv }}$ may in fact be derived from DW, Inv, and DC.

One may appreciate the geometric intuition behind the rule DC if one realizes that the left branch requires one to show that the set of states satisfying $C$ is an invariant for the system initialized in any state satisfying $F$. Thus, the system does not admit any trajectories starting in $F$ that leave $C$ and hence by adding $C$ to the evolution constraint, one does not restrict the behavior of the original system.

Differential cuts may be applied repeatedly to the effect of refining the evolution constraint with more invariant sets. It may be profitable to think of successive differential cuts as showing an embedding of invariants in a system.

There is an interesting connection between differential cuts and embeddings of invariant sub-manifolds, when used with the proof rule Lie. To develop this idea, let us remark that if one succeeds at proving invariance of some $h_{1}=0$ using the rule Lie in a system with no evolution constraint, one shows that $h_{1}=0$ is a smooth invariant submanifold of $\mathbb{R}^{n}$. If one now considers the system evolving inside that invariant manifold and finds some $h_{2}=0$ which can be proved to be invariant using Lie with $h_{1}=0$ acting as an evolution constraint, then inside the manifold $h_{1}=0, h_{2}=0$ defines an invariant sub-manifold (even in cases when $h_{2}=0$ might not define a sub-manifold of the ambient space $\mathbb{R}^{n}$ ). One can proceed using Lie in this way to look for further embedded invariant sub-manifolds. We will illustrate this idea using a basic example.

Example 10 (Differential cut with Lie). Let the system dynamics be $\boldsymbol{p}=\left(x_{1},-x_{2}\right)$. This system has an equilibrium at the origin, i.e. $\boldsymbol{p}(\mathbf{0})=\mathbf{0}$. Consider an invariant candidate $x_{1}=0 \wedge x_{1}-x_{2}=0$. One cannot use Lie directly to prove the goal

$$
x_{1}=0 \wedge x_{1}-x_{2}=0 \rightarrow[\dot{\boldsymbol{x}}=\boldsymbol{p}]\left(x_{1}=0 \wedge x_{1}-x_{2}=0\right) .
$$

Instead, DC can be used to cut by $x_{1}=0$, which is an invariant for this system provable using Lie. The left branch of DC is proved as follows:

$$
\begin{array}{cc}
(\mathbb{R}) \frac{*}{x_{1}=0 \wedge x_{1}-x_{2}=0 \rightarrow x_{1}=0} & (\text { Lie }) \frac{*}{x_{1}=0 \rightarrow x_{1}=0 \wedge(1 \neq 0)} \\
x_{1}=0 \wedge x_{1}-x_{2}=0 \rightarrow\left[\dot{\boldsymbol{x}}=\boldsymbol{p} \& x_{1}=0\right] x_{1}=0 & \frac{\left.x_{1}=\boldsymbol{p}\right] x_{1}=0}{x_{1}}
\end{array}
$$



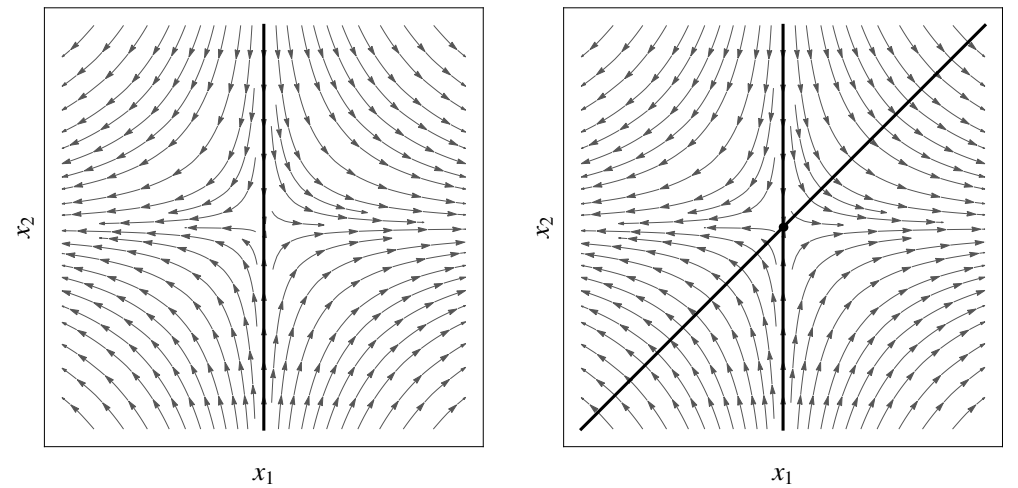

Fig. 2: System invariant $x_{1}=0$ (left) used in a differential cut to show that the intersection at the origin (right) is an invariant.

One can also prove that $x_{1}=x_{2}$ is a invariant under the evolution constraint $x_{1}=0$ :

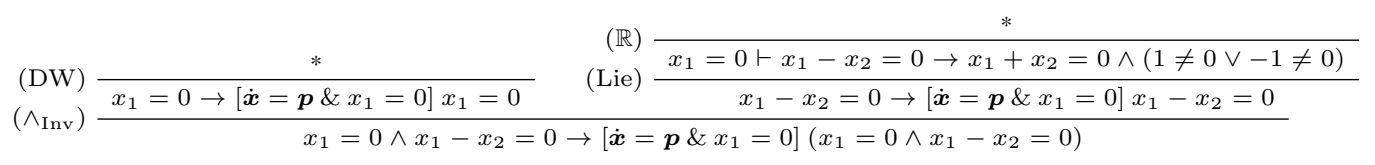

Using these two sub-proofs to close the appropriate branches, the rule DC proves

$$
x_{1}=0 \wedge x_{1}-x_{2}=0 \rightarrow[\dot{\boldsymbol{x}}=\boldsymbol{p}]\left(x_{1}=0 \wedge x_{1}-x_{2}=0\right) .
$$

While this example is very simplistic, it provides a good illustration of the method behind differential cuts. We used DC to restrict system evolution to an invariant manifold $x_{1}=0$ using Lie and then used Lie again to show that $x_{1}-x_{2}=0$ defines an invariant sub-manifold inside $x_{1}=0$. This is illustrated in Fig.2

It is also worth noting that the choice of conjunct for use in the differential cut was crucial. Had we initially picked $x_{1}-x_{2}=0$ to act as $C$ in DC, the proof attempt would have failed, since this does not define an invariant sub-manifold of $\mathbb{R}^{2}$ (see Fig.2).

Let us now remark that by employing DC, we proved invariance of a conjunction which could not be described by an atomic equational assertion which is provable using the rule Lie, or by using Lie to prove invariance of each conjunct after breaking down the conjunction with the rule $\wedge_{\text {Inv }}$. It has previously been shown that differential cuts increase the deductive power of the system when used in concert with differential invariants $[23|26| 25]$. We prove that the same is true for differential cuts with Lie. Indeed, differential cuts serve to address some of the limitations inherent in both $\mathrm{DI}_{=}$and Lie.

Theorem 11. The deductive power of Lie together with DC is strictly greater than that of Lie considered separately. We write this as DC $+\mathrm{Lie} \succ$ Lie.

Proof. In Example 10 we demonstrate the use of Lie together with DC to prove invariance of a conjunction of polynomial equalities which is not provable using Lie 
alone. To see this, suppose that for the system in Example 10 there exists some realvalued differentiable function $g(\boldsymbol{x})$ whose zero level set is precisely the origin, i.e. $(g(\boldsymbol{x})=0) \equiv(\boldsymbol{x}=\mathbf{0})$. Then, for all $\boldsymbol{x} \in \mathbb{R}^{2} \backslash\{\mathbf{0}\}$ this function evaluates to $g(\boldsymbol{x})>0$ or $g(\boldsymbol{x})<0$ (by continuity of $g(\boldsymbol{x})$ ) and $\mathbf{0}$ is thus the global minimum or global maximum, respectively. In either case, $g(\boldsymbol{x})=0 \Longrightarrow \nabla g(\boldsymbol{x})=\mathbf{0}$ is valid, which cannot satisfy the premise of Lie.

Similar to the embedding of invariants observed when combining differential cuts with Lie proof rule, we briefly explore an intriguing connection between the use of differential cuts together with $\mathrm{DI}_{=}$and higher integrals of dynamical systems.

The premise of the rule $\mathrm{DI}=$ establishes that $h(\boldsymbol{x})$ is a first integral (i.e. a constant of motion) for the system in order to conclude that $h=0$ is an invariant. More general notions of invariance have been introduced to study integrability of dynamical systems. For instance, $h(\boldsymbol{x})$ is a second integral if $\mathfrak{L}_{p}(h)=\alpha h$, where $\alpha$ is some function; this is also sufficient to conclude that $h=0$ is an invariant. Let us remark that in a purely polynomial setting, such an $h \in \mathbb{R}[\boldsymbol{x}]$ is known as a Darboux polynomial [13|7] and the condition corresponds to ideal membership in the premise of P-c. Going further, a third integral is a function $h(\boldsymbol{x})$ that remains constant on some level set of a first integral $g(\boldsymbol{x})$ [13, Section 2.6], i.e. $\mathfrak{L}_{\boldsymbol{p}}(h)=\alpha g$ where $g$ is a first integral and $\alpha$ is some function. These ideas generalize to higher integrals (see [13, Section 2.7]).

Example 12 (Deconstructed aircraft [25] - differential cut with $\mathrm{DI}_{=}$). Consider the system $\dot{\boldsymbol{x}}=\boldsymbol{p}=\left(-x_{2}, x_{3},-x_{2}\right)$ and consider the invariant candidate $x_{1}^{2}+x_{2}^{2}=1 \wedge x_{3}=$ $x_{1}$. One cannot use $\mathrm{DI}_{=}$directly to prove the goal

$$
x_{1}^{2}+x_{2}^{2}=1 \wedge x_{3}=x_{1} \rightarrow[\dot{\boldsymbol{x}}=\boldsymbol{p}]\left(x_{1}^{2}+x_{2}^{2}=1 \wedge x_{3}=x_{1}\right) .
$$

We can apply DC to cut by $x_{1}=x_{3}$, which is a first integral for the system and is thus provable using $\mathrm{DI}_{=}$. The left branch of $\mathrm{DC}$ is proved as follows:

$$
(\mathbb{R}) \frac{*}{x_{1}^{2}+x_{2}^{2}=1 \wedge x_{3}=x_{1} \rightarrow x_{3}=x_{1}} \quad\left(\mathrm{DI}_{=}\right) \frac{(\mathbb{R}) \frac{*}{-x_{2}=-x_{2}}}{x_{1}^{2}+x_{2}^{2}=1 \wedge x_{3}=x_{1} \rightarrow[\dot{\boldsymbol{x}}=\boldsymbol{p}] x_{3}=x_{1}}
$$

For the right branch of DC we need to show that $x_{1}^{2}+x_{2}^{2}=1$ is an invariant under the evolution constraint $x_{3}=x_{1}$. This is again provable using $\mathrm{DI}_{=}$:

$$
\left(\wedge_{\text {Inv }}\right) \frac{*}{\frac{x_{3}=x_{1} \rightarrow\left[\dot{\boldsymbol{x}}=\boldsymbol{p} \& x_{3}=x_{1}\right] x_{3}=x_{1}}{x_{1}^{2}+x_{2}^{2}=1 \wedge x_{3}=x_{1} \rightarrow\left[\dot{\boldsymbol{x}}=\boldsymbol{p} \& x_{3}=x_{1}\right]\left(x_{1}^{2}+x_{2}^{2}=1 \wedge x_{3}=x_{1}\right)} \quad\left(\mathrm{DI}_{=}\right) \frac{(\mathbb{R}) \frac{*}{x_{3}=x_{1} \vdash-2 x_{1} x_{2}+2 x_{2} x_{3}=0}}{x_{1}^{2}+x_{2}^{2}=1 \rightarrow\left[\dot{\boldsymbol{x}}=\boldsymbol{p} \& x_{3}=x_{1}\right] x_{1}^{2}+x_{2}^{2}=1}}
$$

We can now construct a proof of invariance for the conjunction using DC.

Note that in this example, we have only ever had to resort to the rule $\mathrm{DI}=$ for showing invariance of an equational candidate. We first showed that $x_{3}-x_{1}$ is an invariant function (first integral) for the system. After restricting the evolution domain to the zero set of the first integral, $x_{3}-x_{1}=0$, we proved that the polynomial $x_{1}^{2}+x_{2}^{2}-1$ is conserved in the constrained system. In this example we have 
$\mathfrak{L}_{p}\left(x_{1}^{2}+x_{2}^{2}-1\right)=-2 x_{1} x_{2}+2 x_{2} x_{3}=2 x_{2}\left(x_{3}-x_{1}\right)$, where $\left(x_{3}-x_{1}\right)$ is a first integral of the system. Thus, $x_{1}^{2}+x_{2}^{2}-1$ is in fact a (polynomial) third integral.

\subsection{Proof Strategies using Differential Cuts}

Differential cuts can be used to search for a proof of invariance of conjunctive equational assertions. This involves selecting some conjunct $h_{i}=0$ to cut by (that is use it as $C$ in DC). If the conjunct is indeed an invariant, it will be possible to strengthen the evolution domain constraint and proceed in a similar fashion by selecting a new $C$ from the remaining conjuncts until a proof is attained. A formal proof of invariance using differential cuts can be quite long and will repeatedly resort to proof rules such as $\left(\wedge_{\text {Inv }}\right)$ (Eq. 12) $)$ and DW (Fig.11), which is used to prune away conjuncts that have already been added to the evolution domain constraint.

Our proof strategy iteratively selects a conjunct with which to attempt a differential cut as a recursive function (DCSearch, elaborated in [12]). Before calling this function, the conjuncts are put into ascending order with respect to the number of variables appearing in the conjunct. For purely polynomial problems, the ordering is also ascending with respect to the total degree of the polynomials. The aim of this pre-processing step is to ensure that conjuncts which are potentially less expensive to check for invariance are processed first (see Section 3.2. There is in general no easy way of selecting the "right" proof rule for showing invariance of atomic equations; a possible, albeit not very efficient, solution would be to iterate through all the available proof rules. This would combine their deductive power, but could also lead do diminished performance. In practice, selecting a good proof rule for atomic invariants is very much a problem-specific matter. We have implemented DCSearch to use the proof rule $\mathrm{DI}=$ before trying Lie.

\subsection{Performance and Limitations}

Unlike with purely automated methods, such as $\mathrm{DRI}_{\wedge}$, knowledge about the system is often crucial for differential cuts to be effective; however, this knowledge can sometimes be used to construct proofs that are more computationally efficient. We have identified an example — detailed in [12]—with 13 state variables which defeats the current implementation of DRI $\wedge$ and which is easily provable using differential cuts together with both $\mathrm{DI}_{=}$and Lie (solved quickly by running DCSearch). Though very much an artificial problem, it demonstrates that structure in the problem can sometimes be exploited to yield efficient proofs using DC. This is especially useful for large systems with many variables where the structure of the problem is well-understood. Additionally, we see that a combination of proof rules $\left(\mathrm{DI}_{=}, \mathrm{Lie}, \mathrm{DC}\right)$ can be both helpful and efficient.

While differential cuts can serve to increase the deductive power of sufficient proof rules, there are invariant conjunctions of equalities for which applying DC on the conjuncts given in the problem will altogether fail to be fruitful. This is due to DCSearch relying on the fact that at least some of the conjuncts considered individually are invariants for the system, which may not be the case even if the conjunction is invariant. 


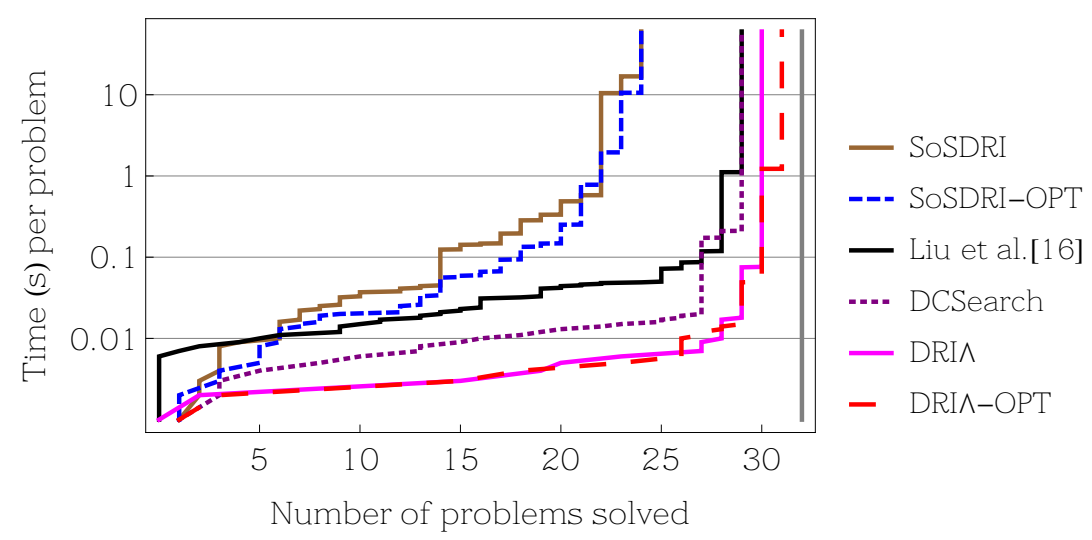

Fig. 3: Empirical performance comparison of different proof rules and strategies. The total number of problems solved each in at most $t$ s (log scale) is given in the $x$-axis for each method.

\section{Experiments}

In this section, we empirically compare the performance of three families of proof rules for checking the invariance of conjunctions: (1) DRI-related proof rules including SoSDRI (DRI plus sum-of-squares rewriting), $\mathrm{DRI}_{\wedge}$ as well as their optimized versions as detailed in Section 3.3. (2) DCSearch: the differential cut proof search presented in Section 5.1, and (3) the Liu et al. procedure [16] applied to a conjunction of equalities.

We do not consider domain constraints, i.e. $H=$ True. The running time for each proof rule as well as the dimension, the different degrees of the candidates and the vector fields, of the used set of benchmarks can be found in the companion report [12]. In Fig. 3, the pair $(k, t)$ in the plot of a proof rule $P$ reads: the proof rule $P$ solved $k$ problems each in less than $t$ seconds. The set of benchmarks contains 32 entries composed of equilibria (16), singularities (8), higher integrals (4) and abstract examples (4). The examples we used in our benchmarks originate from a number of sources - many of them come from textbooks on Dynamical Systems; others have been hand-crafted to exploit sweetspots of certain proof rules. For instance, we constructed Hamiltonian systems, systems with equilibria and systems with smooth invariants of various polynomial degrees. The most involved example has 13 state variables, a vector field with a maximum total degree of 291 and an invariant candidate with total degree of 146. It should be noted that these benchmarks are not necessarily representative, but nevertheless, an important first step towards a more comprehensive empirical analysis we hope to pursue.

One can clearly see that for the considered set of examples, the proof rule $\mathrm{DRI}_{\wedge}$ is much more efficient on average compared to SoSDRI as it solves 31-out of 32-in less than $0.1 \mathrm{~s}$ each. The optimization discussed in Section 3.3 yields a slight improvement in the performance of both SoSDRI and DRI $\wedge$. Notice that its benefit is clearer in SoSDRI as the involved polynomials have large degrees. In most examples, both $\mathrm{DRI}_{\wedge}$ 
and $\mathrm{DRI}_{\wedge}-\mathrm{OPT}$ are very efficient. However, the optimized version was able to falsify, in $1.2 \mathrm{~s}$, an invariant whereas the unoptimized version, as well as all the other proof rules, timed out after 60s. We also noticed for another example-featuring the Motzkin polynomial-that SoSDRI-OPT timed out whereas SoSDRI was able to check the invariance in $15 \mathrm{~s}$. When we investigated this example, it turned out that the rational coefficients of the remainder gets complicated compared to the original polynomial before reduction. For this particular example, the optimized version was able to prove the invariance in 300s which is 20 times slower than the unoptimized version. For a third example, all DRI-related proof rules timed out after 60 s in one example which was discharged by DCSearch in less than 6s. (cf. [12] for more details about those different examples).

\section{Related Work}

In this paper we focus on checking invariance of algebraic sets under the flow of polynomial vector fields. For similar techniques used to automatically generate invariant algebraic sets we refer the reader to the discussion in [11].

Nagumo's Theorem [3], proved by Mitio Nagumo in 1942, characterizes invariant closed sets-a superset of algebraic sets-of locally Lipschitz-continuous vector fields-a superset of polynomial vector fields. The geometric criterion of the theorem is however intractable. The analyticity of solutions of analytic vector fields-a superset of polynomial vector fields-also gives a powerful, yet intractable, criterion to reason about invariant sets. In [28], the authors attempted to define several special cases exploiting either Nagumo's theorem or the analyticity of solutions, to give proof rules for checking invariance of (closed) semi-algebraic sets under the flow of polynomial vector fields. Liu et al. in [16] also used analyticity of solutions to polynomial ordinary differential equations and extended [28] using the ascending chain condition in Noetherian rings to ensure termination of their procedure; they gave a necessary and sufficient condition for invariance of arbitrary semi-algebraic sets under the flow of polynomial vector fields and proved the resulting conditions to be decidable.

We develop a purely algebraic approach where the ascending chain condition is also used but without resorting to local Taylor series expansions. As in [16], we require finitely many higher-order Lie derivatives to vanish; what is different, however, is the definition of the finite number each characterization requires: in [16], one is required to compute orders $N_{i}$ of each atom $h_{i}$ and to prove that all higher-order Lie derivatives of $h_{i}$, up to order $N_{i}-1$, vanish. We state a weaker condition as we only require that all higher-order Lie derivatives of $h_{i}$ up to order $(N-1)$, for all $i$, vanish. A straightforward benefit of our characterization is the immediate reduction of the computational complexity as discussed in Section 3 and shown empirically in Section 6 .

Zerz and Walcher [30] have previously considered the problem of deciding invariance of algebraic sets in polynomial vector fields; they gave a sufficient condition for checking invariance of algebraic sets which can be seen as one iteration of Algorithm 1 . Therefore, Section 3 generalizes their work by providing a complete characterization of invariant algebraic sets in polynomial vector fields. 


\section{Conclusion}

We have introduced an efficient decision procedure $\left(\mathrm{DRI}_{\wedge}\right)$ for deciding invariance of conjunctive equational assertions for polynomial dynamical systems. We have explored the use of the differential cut rule both as a means of increasing the deductive power of existing sufficient proof rules and also as a way of constructing more computationally efficient proofs of invariance.

The empirical performance we observe in the optimized implementations of DRI and $\mathrm{DRI}_{\wedge}$ is very encouraging and we are confident that a proof strategy in a deductive formal verification system should give precedence to these methods. However, certain problems fall out of scope of these rules. For instance, when the problems involve transcendental functions, or still take unreasonably long time to prove. We leave these interesting questions for future work.

\section{References}

1. Basu, S., Pollack, R., Roy, M.F.: On the combinatorial and algebraic complexity of quantifier elimination. J. ACM 43(6), 1002-1045 (1996)

2. Bayer, D., Stillman, M.E.: A criterion for detecting m-regularity. Inventiones Mathematicae 87, 1 (1987)

3. Blanchini, F.: Set invariance in control. Automatica 35(11), 1747-1767 (1999)

4. Buchberger, B.: Gröbner-Bases: An Algorithmic Method in Polynomial Ideal Theory. Reidel Publishing Company, Dodrecht - Boston - Lancaster (1985)

5. Collins, G.E., Hong, H.: Partial cylindrical algebraic decomposition for quantifier elimination. J. Symb. Comput. 12(3), 299-328 (1991)

6. Cox, D.A., Little, J., O'Shea, D.: Ideals, Varieties, and Algorithms - an introduction to computational algebraic geometry and commutative algebra (2. ed.). Springer (1997)

7. Darboux, J.G.: Mémoire sur les équations différentielles algébriques du premier ordre et du premier degré. Bulletin des Sciences Mathématiques et Astronomiques 2(1), 151-200 (1878)

8. Dubé, T.: The structure of polynomial ideals and Gröbner bases. SIAM J. Comput. 19(4), 750-773 (1990)

9. Faugère, J.C.: A new efficient algorithm for computing Gröbner bases (F4) . Journal of Pure and Applied Algebra 139(13), 61 - 88 (1999)

10. Faugère, J.C.: A new efficient algorithm for computing Gröbner bases without reduction to zero (F5). In: Proceedings of the 2002 International Symposium on Symbolic and Algebraic Computation. pp. 75-83. ISSAC '02, ACM, New York, NY, USA (2002)

11. Ghorbal, K., Platzer, A.: Characterizing algebraic invariants by differential radical invariants. In: Ábrahám, E., Havelund, K. (eds.) TACAS. LNCS, vol. 8413, pp. 279-294. Springer (2014)

12. Ghorbal, K., Sogokon, A., Platzer, A.: Invariance of conjunctions of polynomial equalities for algebraic differential equations. Tech. Rep. CMU-CS-14-122, School of Computer Science, CMU, Pittsburgh, PA (6 2014), http: //reports-archive.adm.cs . cmu . edu/anon/2014/abstracts/14-122.html

13. Goriely, A.: Integrability and Nonintegrability of Dynamical Systems. Advanced series in nonlinear dynamics, World Scientific (2001)

14. Lazard, D.: Gröbner-bases, Gaussian elimination and resolution of systems of algebraic equations. In: van Hulzen, J.A. (ed.) EUROCAL. LNCS, vol. 162, pp. 146-156. Springer (1983) 
15. Lie, S.: Vorlesungen über continuierliche Gruppen mit Geometrischen und anderen Anwendungen. Teubner, Leipzig (1893)

16. Liu, J., Zhan, N., Zhao, H.: Computing semi-algebraic invariants for polynomial dynamical systems. In: Chakraborty, S., Jerraya, A., Baruah, S.K., Fischmeister, S. (eds.) EMSOFT. pp. 97-106. ACM (2011)

17. Matringe, N., Moura, A.V., Rebiha, R.: Generating invariants for non-linear hybrid systems by linear algebraic methods. In: Cousot, R., Martel, M. (eds.) SAS. LNCS, vol. 6337, pp. 373-389. Springer (2010)

18. Mayr, E.W.: Membership in polynomial ideals over Q is exponential space complete. In: Monien, B., Cori, R. (eds.) STACS. LNCS, vol. 349, pp. 400-406. Springer (1989)

19. Mayr, E.W., Meyer, A.R.: The complexity of the word problems for commutative semigroups and polynomial ideals. Advances in Mathematics 46(3), 305 - 329 (1982)

20. Neuhaus, R.: Computation of real radicals of polynomial ideals II. Journal of Pure and Applied Algebra 124(13), 261 - 280 (1998)

21. Olver, P.J.: Applications of Lie Groups to Differential Equations. Springer (2000)

22. Platzer, A.: Differential dynamic logic for hybrid systems. J. Autom. Reasoning 41(2), 143 189 (2008)

23. Platzer, A.: Differential-algebraic dynamic logic for differential-algebraic programs. J. Log. Comput. 20(1), 309-352 (2010)

24. Platzer, A.: Logical Analysis of Hybrid Systems - Proving Theorems for Complex Dynamics. Springer (2010)

25. Platzer, A.: A differential operator approach to equational differential invariants - (invited paper). In: Beringer, L., Felty, A.P. (eds.) ITP. LNCS, vol. 7406, pp. 28-48. Springer (2012)

26. Platzer, A.: The structure of differential invariants and differential cut elimination. Logical Methods in Computer Science 8(4), 1-38 (2012)

27. Sankaranarayanan, S., Sipma, H.B., Manna, Z.: Constructing invariants for hybrid systems. Formal Methods in System Design 32(1), 25-55 (2008)

28. Taly, A., Tiwari, A.: Deductive verification of continuous dynamical systems. In: Kannan, R., Kumar, K.N. (eds.) FSTTCS. LIPIcs, vol. 4, pp. 383-394. Schloss Dagstuhl - LeibnizZentrum fuer Informatik (2009)

29. Tarski, A.: A decision method for elementary algebra and geometry. Bulletin of the American Mathematical Society 59 (1951)

30. Zerz, E., Walcher, S.: Controlled invariant hypersurfaces of polynomial control systems. Qualitative Theory of Dynamical Systems 11(1), 145-158 (2012) 